\title{
The Two Primary Tasks on the Way to National e_Learn Grid Node
}

\author{
V. Reklaitis \\ Graduate School of Information Systems, \\ University of Electro-Communications, Tokyo, Japan \\ vytas@is.uec.ac.jp \\ K. Baniulis, A. Masevicius \\ Computer Networking Department, \\ Kaunas University of Technology, Lithuania \\ kazysba@pit.ktu.It zolia@lydys.sc-uni.ktu.It
}

\begin{abstract}
E_learning as a class of applications can benefit from Grid service-oriented computing. In this short paper we focus on technological and educational issues of using Grid technologies for e-learning from the perspective of a Lithuanian national grid node at Kaunas University of Technology.
\end{abstract}

The immense growth of interest in the Grid computing uplifted the rethinking the Grid concept itself [1]. The renewed understanding of the Grid may be interpreted as a deviation from the static Grid definition based on the three layer classification - computation, information, knowledge. According to the new vision Grid is considerably more dynamic entity focused on providing of nontrivial services through resource sharing and communication. Grid is also seen as service oriented infrastructure in the Report [2].

On the other hand, such an accent on services and applications could be considered as a fact that grid technology has already reached a certain level of maturity, especially as regards computational and data processing grids. In these two areas the grid technology is even driven behind by a new research and business applications. But different situation is in e-learning area where content objects predominate, they can be retrieved from repository and copied into the instance of webbased lesson or entire course. Such applications are successfully deployed using popular learning environments WebCT, Blackboard. Generally speaking, e_learning area still does not have applications adequate to Grid and in this respect the Grid technology is leading e_learning application development visibly.

The LeGE-WG aims at facilitating collaboration between European partners within two, at the first glance, distinctive fields - Grid technology and e_learning. We say distinctive because Grid technology is a distributed systems and computing at large including large scale networking, while a great majority of e_learning applications currently in use can be attributed to the category of rather simple client-server applications. Therefore the LeGE-WG objectives, from our point of view, are to bridge the gap between the Grid technology and e learning in many ways by consolidating research in order to achieve a commonly acceptable in-depth understanding of integrated e_learning Grid infrastructure.

In this short paper we focus on technological and educational issues of using Grid technologies for e_learning from the perspective of a Lithuanian national grid node at Kaunas University of Technology (KTU). We envisage two primary tasks:

1. To establish the advanced network and hosting environment including necessary executing facilities, a certain Grid software platform and other toolkits for grid services development, provision and management, 
2. To find out at least a few University level e_learning applications relevant to Grid technology and take them for pilot realization as grid-aware applications within our hosting environment.

From the perspective of the network technology for grid services provision, the most advanced network in our country is LitNET - National Research and Education Network (NREN) and Network Operating Centre (NOC) housed at KTU. LitNET interconnects about 450 educational and research institutions, including all 15 universities, as well colleges and secondary schools across the country. Currently all universities have $1 \mathrm{Gbps}$ access to the core network, data transport service is being provided using IPv6, multicast. LITNET is linked to the high capacity pan-European network GEANT via $155 \mathrm{Mbps}$ channel, which is going to be upgraded up to $655 \mathrm{Mbps}$ in first half of 2003. Accordingly, in order to accept and distribute data streams at GEANT speed the significant changes in the internal LitNET infrastructure are taking place too. The LitNET infrastructure and resources make grid level services technologically feasible.

Setting up a hosting environment to examine and experiment with software toolkits aiming to estimate their usability for establishment of rather specific e_learning services is another part of the first task. Among grids the Globus technological framework is the first candidate to work with because it is going to be implemented completely based on Open Grid Service Architecture specification [3]. E_learning is a class of applications that can benefit from Grid service-oriented computing. But it is always useful to be ascertained what kind of means a framework comprises for effective development and deployment of highly interactive e_learning applications on a Grid. In this respect, we look forward to sharing knowledge and expertise among LeGE-WG partners as a mechanism facilitating the adoption of Grid technology and software platform for Grid-aware e_learning solutions.

Design, development and setting up of e_learning applications relevant to the Grid technology is the second task of primary importance and must be discussed within LeGE-WG. From the perspective of learning service provision not only grid technology or software platform should be considered, but also pedagogically proven e_learning solutions for various categories of learners. Actually we are driven by users - teachers and learners. The success in exploitation of Grid technology will greatly depend on methodologically excellent examples of e_learning applications ready to use on the Grid. In particular, the intended grid applications must demonstrate their distinctive features with respect to the Grid environment. Grid category of services until now is seen mainly as computational and data processing services that cannot be provided by a single machine. We must worry about how highly interactive e_learning services, where activities are sequenced depending on specified learner's actions, will fit into Grid service provision architecture.

In answer to EU call to create information society in the country there are growing support for network-based educational developments. On the other hand, there is a diverse e-learning audience. As a national grid node we are going to focus on analysis and selection of grid relevant e_learning applications, which differ from conventional e_learning courseware by large scale resource and/or data sharing while performing simulations and calculations. This category of applications is more typical to higher education study programmes, including studies and research when seeking for MSc and PhD degrees. For instance, a project with participation of several Lithuanian universities is going to be initiated to interconnect and to make interoperable educational resources for MSc and PhD study programmes. These resources as software products inherently are more complex, include calculations and simulations, and therefore more typical to be used on grid. Naturally e_learning application put on a grid as a service should be suitable not only for one university. Therefore WG forum could help to sort out applications to be implemented at first for research and practical validation of both technology and didactical effectivenness.

In conclusion, Lithuanian partner KTU is well positioned to play the role of the national grid node. We are seeking for a reasonable balance between technology and applications. The University is the leading e_learning institution in the country and has developed considerable expertise in the design of e_learning settings and applications. The co-existence and close collaboration between 
the University and LITNET NOC guarantees support for both building of grid infrastructure and carrying out of learning experiments.

\section{REFERENCES}

Baker, M., Buyya, R., Laforenza D., (2002) Grids and Grid technologies for wide-area distributed computing. Software Practice \& Experience, 32, 1437-1466.

De Roure, D.,Jennings, N., Shadbolt, N., (2001) Research Agenda for the Semantic GRID: a Future e-Science Infrastructure. Report commissioned for EPSRC/DTI Core e-Science Programme http://www.semanticgrid.org/v1.9/semgrid.pdf (accessed Febr 2003)

Foster. I. (2002) What is the Grid? A Three Point Checklist http://www.gridtoday.com/02/0722/100136.html (accessed Febr 2003) 